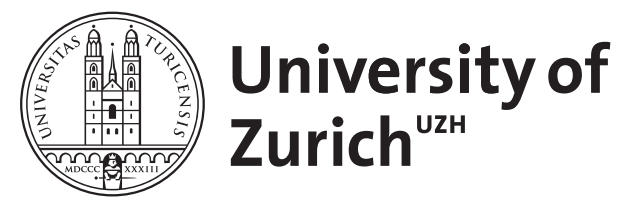

\title{
Spinal pain due to metastasis of unknown origin
}

Stoll, Susanna ; Taverna, Christian ; Michel, Beat A ; Sprott, Haiko

\begin{abstract}
A 58-year-old woman presented with non-radicular pain in the upper thorax. Due to the prolonged duration of symptoms, a bone scintigraphy was made, which showed pathological enhancement in the upper thoracic spine. An MRI demonstrated lesions of the third and fourth thoracic vertebrae. A biopsy showed a metastasis of poorly differentiated carcinoma. A whole-body 18-F-FDG PET scan failed to identify a primary tumour. The patient was given radiotherapy, chemotherapy and analgesic treatment. She died within 3years. In the late stage, the tumour marker CA 19-9 was positive; however, an MRI of the abdomen failed to identify a pancreatic tumour. Metastasis from an unknown primary site can present as cervical spinal disease very similar to degenerative disease
\end{abstract}

DOI: https://doi.org/10.1007/s00296-004-0575-5

Posted at the Zurich Open Repository and Archive, University of Zurich ZORA URL: https://doi.org/10.5167/uzh-155976

Journal Article

Published Version

Originally published at:

Stoll, Susanna; Taverna, Christian; Michel, Beat A; Sprott, Haiko (2005). Spinal pain due to metastasis of unknown origin. Rheumatology international, 25(7):562-564.

DOI: https://doi.org/10.1007/s00296-004-0575-5 


\section{Susanna Stoll $\cdot$ Christian Taverna $\cdot$ Beat A. Michel \\ Haiko Sprott \\ Spinal pain due to metastasis of unknown origin}

Received: 17 September 2004 / Accepted: 11 October 2004/Published online: 2 February 2005

(C) Springer-Verlag 2005

\begin{abstract}
A 58-year-old woman presented with nonradicular pain in the upper thorax. Due to the prolonged duration of symptoms, a bone scintigraphy was made, which showed pathological enhancement in the upper thoracic spine. An MRI demonstrated lesions of the third and fourth thoracic vertebrae. A biopsy showed a metastasis of poorly differentiated carcinoma. A wholebody 18 -F-FDG PET scan failed to identify a primary tumour. The patient was given radiotherapy, chemotherapy and analgesic treatment. She died within 3 years. In the late stage, the tumour marker CA 19-9 was positive; however, an MRI of the abdomen failed to identify a pancreatic tumour. Metastasis from an unknown primary site can present as cervical spinal disease very similar to degenerative disease.
\end{abstract}

Keywords Pain $\cdot$ Skeletal $\cdot$ Metastasis $\cdot$ PET

\section{Introduction}

Cervical spinal pain is a common symptom in patients with degenerative changes of the cervical spine or myofascial pain syndrome. An X-ray is not necessarily

This revised version was published online in March 2005 with corrections to Figure 1.

S. Stoll $\cdot$ C. Taverna

Department of Oncology,

University Hospital Zurich,

Rämistrasse 100, 8091 Zurich,

Switzerland

B. A. Michel · H. Sprott ( $\square)$

Department of Rheumatology and Institute of Physical Medicine, University Hospital Zurich,

Gloriastrasse 25, 8091 Zurich,

Switzerland

E-mail: haiko.sprott@usz.ch

Tel.: +41-44-2553010

Fax: + 41-44-2554388 the first examination if no red flag [1] is present. Usually, the patients in our department are treated with NSAIDs, myorelaxants and/or physical therapy and followed up carefully. Laboratory tests and X-rays are performed only if the patient's condition does not improve within 2-4 weeks.

\section{Case report}

A 58-year-old woman was admitted to our university hospital in September 2000 with a 10-month history of back pain in the upper thorax that did not respond well to physiotherapy or systemic analgesics. Physical examination revealed an afebrile, obese (BMI $35.7 \mathrm{~kg}$ / $\mathrm{m}^{2}$ ) patient with normal findings except a slight pain on percussion of the third and fourth thoracic vertebrae. Laboratory tests (ESR, CRP, electrolytes, liver enzymes and creatinine) were normal. Lactate dehydrogenase (LDH) was not determined. The whole-body technetium-99 scintigraphy showed a massive focal uptake in the upper part of the thoracic vertebral column. In addition, an MRI of the cervical and thoracic spine disclosed increased signal intensity of the third and fourth thoracic vertebrae with an intense uptake of contrast media in the contiguous soft tissue (Fig. 1a). A CT scan of the neck, chest and abdomen and an MRI of the abdomen showed a lobular cystic lesion in the right liver lobe but no signs for primary tumour. An $18 \mathrm{~F}$ FDG PET confirmed the high FDG uptake of the third and fourth thoracic vertebrae (Fig. 1d). The lesion of the right liver lobe showed only a low uptake and was thought to be a cyst.

Since a CT-guided bone biopsy of the third thoracic vertebra showed only necrotic bone tissue, open biopsy was performed. It revealed metastasis from poorly differentiated carcinoma of unknown origin. The neoplastic cells stained positive for cytokeratin and S100 but were negative for the oestrogen and progesterone receptor. Other tumour markers (AFP, CEA, CA125, CA15-3, CA19-9) were negative at this time. 
Fig. 1 Osteoplastic lesions of the third and fourth thoracic vertebrae on MRI

(a), conventional X-ray (b) and CT scan (c). Pathological findings are indicated with asterisks. 18-F-FDG PET scans (d) revealed high FDG uptake in the third thoracic vertebra (red spots)
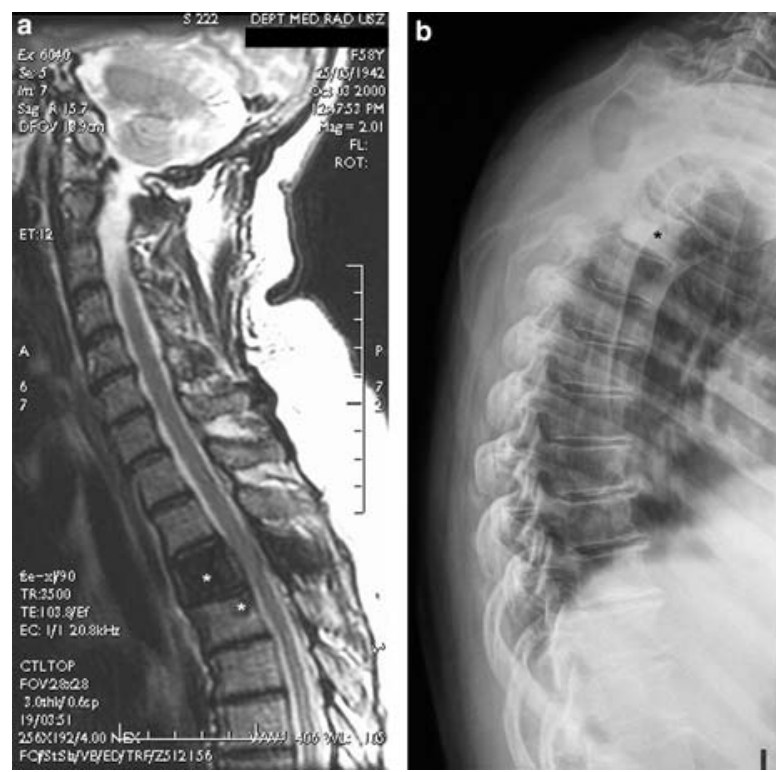
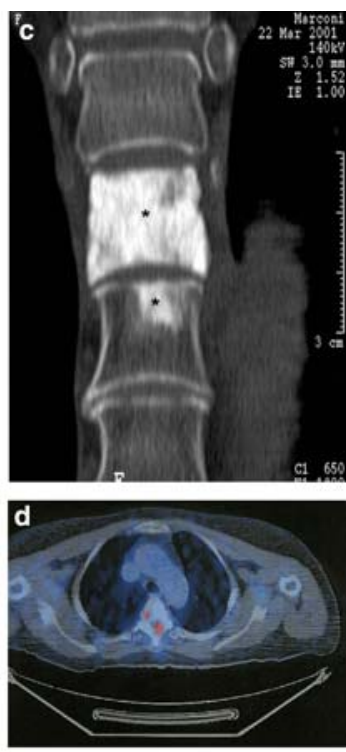

The patient received local percutaneous radiotherapy with $20 \times 2$ Gy (totally 40 Gy) from November 2000 to December 2000 with a curative intent [2]. Her symptoms diminished under therapy. Seven months later, she suffered from back pain again at the same thoracic level. A PET scan revealed a high FDG uptake in the third thoracic vertebra and additionally in the os sacrum and a left supraclavicular lymph node. The cystic lesion in the right liver lobe was larger than at the earlier examination with a focal FDG uptake at the margins. It was now assumed to be a cystic metastasis. In view of the clearly visible tumour progression, we decided to start chemotherapy with paclitaxel $\left(175 \mathrm{mg} / \mathrm{m}^{2}\right)$ and carboplatin (AUC 6) as an intravenous infusion every 3 weeks. The PET scan after three courses showed no further FDG uptake, and thus a complete remission was postulated. A total of six courses were given.

In May 2002, 2 months after the end of the chemotherapy, the patient again complained of considerable pain in the upper back. The patient decided to try a homeopathic procedure, to which she did not respond. Three months later, the back pain worsened and resulted in pain-related immobility. She developed paraplegia with weakness of both legs. An MRI yielded compression of the spinal cord at the level of the third and fourth thoracic vertebrae. The patient was transferred to the Department of Surgery where laminectomy, tumour debulking and dorsal stabilisation were performed. Shortly thereafter, a deep vein thrombosis in the right calf was diagnosed, and therapy with low-molecularweight heparin was started. The backache was the main problem and was finally controlled with hydromorphone. In October 2002, the patient was referred to a rehabilitation clinic. There, a second deep vein thrombosis of the left leg was diagnosed, and an oral anticoagulation with phenprocoumon was started.

In the course of the next 3 months, the patient's mobility deteriorated continuously. At the beginning of
January 2003, she experienced sharp pain and cramps in the upper abdomen. On examination, her liver was markedly enlarged. The laboratory test revealed elevated values of alanine aminotransferase (ALT), alkaline phosphatase, bilirubin and LDH, and for the first time elevated CA 19-9. Four weeks later, she was admitted to the emergency ward of a local hospital because of a sudden exacerbation of pain in the upper abdomen. The INR value was $>7$. An ultrasonography showed a large, partially cystic, partially solid lesion in the liver. An acute haemorrhage from the liver lesion was postulated. The bleeding was thought to be caused by tumour progression and anti-coagulation. Except for pain medication, the patient desired no further therapy. She died 1 day after admission. On request of her family, no autopsy was performed.

\section{Discussion}

Metastatic cancer is among the most frequent causes of skeletal pain in adults [3]. Patients without a known primary carcinoma present a diagnostic dilemma. Prostate, breast and lung neoplasms account for approximately $60 \%$ of the metastasis [4]. Bone scintigraphy is more sensitive than radiography and provides a survey of the entire skeleton. However, increased uptake seen on bone scintigraphy is not specific, and some neoplasms are poorly detected by scintigraphy [3]. In $30 \%$ of patients, the primary carcinomas cannot be identified when bone metastases are first diagnosed [5]. MRI and PET may be helpful in detecting and defining the extent and precise location of bone marrow lesions and the extension of soft tissue neoplasms [6].

Pain could be an important indicator of bone metastasis. Although most patients with bone metastases report bone pain, a significant fraction $(21 \%$ of breast and $22 \%$ of prostate cancer patients) is asymptomatic [7]. 
However, pain is not a reliable indicator of the presence of metastatic bone disease. Metastatic bone disease should be taken into consideration in patients with spinal pain who do not respond well to conventional treatment with NSAIDs, myorelaxants and/or physical therapy.

Acknowledgements The authors wish to thank Leanne Pobjoy for her help in preparing the manuscript.

\section{References}

1. http://www.medinfo.co.uk/conditions/lowbackpain.html

2. Moller T (1996) Skeletal metastases. Acta Oncol 35 (Suppl 7): 125-136
3. Buckwalter JA, Brandser EA (1997) Metastatic disease of the skeleton. Am Fam Physician 55:1761-1768

4. Rao S, Badani K, Schildhauer T, Borges M (1992) Metastatic malignancy of the cervical spine: a nonoperative history. Spine 17 (Suppl 10):S407-S412

5. Shih LY, Chen TH, Lo WH (1992) Skeletal metastasis from occult carcinoma. J Surg Oncol 51:109-113

6. Kitagawa Y, Nishizawa S, Sano K, Ogasawara T, Nakamura M, Sadato N, Yoshida M, Yonekura Y (2003) Prospective comparison of $18 \mathrm{~F}-\mathrm{FDG}$ PET with conventional imaging modalities (MRI, CT, and 67 Ga scintigraphy) in assessment of combined intraarterial chemotherapy and radiotherapy for head and neck carcinoma. J Nucl Med 44:198-206

7. Palmer E, Henrikson B, McKusick K, Strauss HW, Hochberg F (1988) Pain as an indicator of bone metastasis. Acta Radiol 29:445-459 